OPEN ACCESS

Edited by:

Snehlata Jaswal,

LM Thapar School of Management,

India

Reviewed by:

Emily M. Elliott,

Louisiana State University, USA

Rick Thomas,

Georgia Institute of Technology, USA

*Correspondence:

Enrica Donolato

enrica.donolato@gmail.com

Specialty section: This article was submitted to

Cognitive Science,

a section of the journal

Frontiers in Psychology

Received: 12 July 2016

Accepted: 12 April 2017

Published: 04 May 2017

Citation:

Donolato $E$, Giofrè $D$ and Mammarella IC (2017) Differences in Verbal and Visuospatial Forward and Backward Order Recall: A Review of the Literature. Front. Psychol. 8:663.

doi: 10.3389/fpsyg.2017.00663

\section{Differences in Verbal and Visuospatial Forward and Backward Order Recall: A Review of the Literature}

\author{
Enrica Donolato ${ }^{1 *}$, David Giofrè ${ }^{2}$ and Irene C. Mammarella ${ }^{1}$ \\ ${ }^{1}$ Department of Developmental and Social Psychology, University of Padova, Padova, Italy, ${ }^{2}$ Department of Natural Sciences \\ and Psychology, Liverpool John Moores University, Liverpool, UK
}

How sequential, verbal and visuospatial stimuli are encoded and stored in memory is not clear in cognitive psychology. Studies with order recall tasks, such as the digit, and Corsi span, indicate that order of presentation is a crucial element for verbal memory, but not for visuospatial memory. This seems to be due to the different effects of forward and backward recall in verbal and visuospatial tasks. In verbal span tasks, performance is worse when recalling things in backward sequence rather than the original forward sequence. In contrast, when it comes to visuospatial tasks, performance is not always worse for a modified backward sequence. However, worse performance in backward visuospatial recall is evident in individuals with weak visuospatial abilities; such individuals perform worse in the backward version of visuospatial tasks than in the forward version. The main aim of the present review is to summarize findings on order recall in verbal and visuospatial materials by considering both cognitive and neural correlates. The results of this review will be considered in the light of the current models of WM, and will be used to make recommendations for future studies.

\footnotetext{
Keywords: order recall, verbal working memory, visuospatial working memory, short-term memory, neural correlates
}

\section{INTRODUCTION}

The ability to process serially ordered information is fundamental to many aspects of our lives, including spelling and orientation to a new environment. However, the cognitive mechanisms underlying encoding and recall of verbal and visuospatial sequences are still not fully understood.

One of the processes involved in serial recall is short-term memory (STM), which allows individuals to hold a small amount of information for a short period of time. Verbal STM is generally tested with the digit span task (DST) that involves recalling sequences of digits, while the ability to retrieve visuospatial information is typically tested with the Corsi span task (CST) that involves recalling sequences of blocks (Berch et al., 1998). In both verbal and visuospatial span tasks, participants may be asked to recall the information in either forward or backward order. In the DST, performance is usually worse in the backward version of the task (Baddeley, 1986; Li and Lewandowsky, 1995), while recall of the forward and backward versions of the CST is much the same for most subjects (Wilde and Strauss, 2002; Cornoldi and Mammarella, 2008). Although, these results give the impression that forward and backward verbal and visuospatial span tasks likely measure different constructs, experimental and neural correlated findings regarding 
serial recall tasks are not consistent, suggesting a need for further research. It is also important to carefully review the research and literature on this subject to-date. Hence, here we will summarize the findings from studies of forward and backward recall in verbal and visuospatial domains as it relates to both cognitive and neural correlates.

\section{Selection of Studies}

We conducted a literature search via PsychINFO, Web of Science and Google Scholar electronic databases. We used the following search keywords: serial/order recall, forward/backward span/recall, DST and CST, verbal/visuospatial, STM/WM. We searched for these terms in titles, in abstracts, and in the keyword lists themselves. Titles and abstracts were screened for appropriateness and independently reviewed for relevance. Papers published from January 1960 to September 2016 have been considered. 132 manuscripts were originally selected for scrutiny; ultimately, only 54 met our inclusion criteria and were considered in the present review.

Papers were considered for inclusion if they covered: (i) behavioral or neural correlates for forward and/or backward recall in the verbal and/or visuospatial domain; and (ii) the impact of verbal and/or visuospatial memory capacity. Studies focusing purely on theoretical models for memory systems were not considered.

\section{Similarities and Differences in Order Recall in Verbal or Visuospatial Domains}

Studies in order recall on verbal and visuospatial domains to-date have used different methodologies and had different aims.

Forward and backward serial position curves have been analyzed by considering primacy and recency effects in verbal WM (Table 1, verbal WM). As for backward recall, findings have shown a qualitative change in serial position curves characterized by an increased recency effect and a decreased primacy effect (Li and Lewandowsky, 1993, 1995; Hulme et al., 1997, see also Penney, 1989 for a review). Likewise, in forward and backward visuospatial tasks, both primacy and recency effects also occur (Farrand and Jones, 1996; Farrand et al., 2001).

Several studies adopted the dual task paradigm when examining verbal tasks. In the literature, the most commonly used examples of dual tasks are articulatory suppression, and irrelevant speech (i.e., concurrent irrelevant sounds). These dual tasks effectively impair memory performance (see Baddeley and Hitch, 1974). In two separate studies, Bireta et al. (2010) and Guérard et al. (2012) explored these effects using similar methods and procedures, but came to different conclusions. In both studies, all the above-mentioned effects were confirmed on forward recall, but only Guérard et al. (2012) found the dual tasks had an impact on backward recall as well. Ritchie et al. (2015) conducted a meta-analysis of 16 experiments focusing on secondary tasks. Irrelevant speech was observed to have a weak effect on task performance. In contrast, articulatory suppression had very large effects, and seemed to disrupt recall for both first and late responses (i.e., primacy and recency), in forward and backward recall alike.
As for the visuospatial domain, studies tend to take one of two approaches: comparing extreme groups, or analyzing clinical populations (Table 1, visuospatial WM). For example, adults with high spatial abilities demonstrated very similar performance in the forward and backward versions of the CST (Wilde and Strauss, 2002), whereas participants with low spatial abilities demonstrated lower performances in backward recall (Cornoldi and Mammarella, 2008). This finding was also confirmed in children with non-verbal learning disability who had severe problems in the spatial domain (Cornoldi et al., 2003; Mammarella and Cornoldi, 2005; Garcia et al., 2014).

Other research directly compared the verbal and visuospatial domains (Table 1, verbal and visuospatial WM) by using the dual task paradigm. Research has shown that a serial secondary task (i.e., spatial tapping) interferes with recall of spatial information (Jones et al., 1995; Vandierendonck et al., 2004). Further, the presence of a verbal secondary task affects both verbal and visuospatial recall when the secondary task requires the manipulation of ordered information. However, when the secondary task requires the manipulation of unordered materials, visuospatial performance is not affected (Depoorter and Vandierendonck, 2009). This result was used by the researchers as evidence of the existence of a cross modal interference. Others argued that the effect seen in Depoorter and Vandierendonck's research was probably due to the specific manipulation used in the study (Logie et al., 2016). However, in further research a different manipulation was employed, and the results confirmed the existence of cross modal interference between verbal and spatial recall performance (Vandierendonck, 2016). This effect was also confirmed using visuospatial materials only (i.e., the CST), showing that both forward and backward recall were affected by the presence of a verbal secondary tasks (Higo et al., 2014).

\section{Neural Correlates}

Although, there is not a general consensus on a specific WM model, neuroscience studies can help to shed further light on the effect of order recall in verbal and visuospatial domains. In fact, event-related potentials (ERPs) and functional Magnetic Resonance Imaging (fMRI) have also been used to investigate neural correlates of forward and backward recall in the verbal and visuospatial domains (see Table 2).

ERPs were used in the backward DST under two conditions; digits were aurally presented and were followed by a second set that either corresponded to the reverse order (correct condition), or by a second set in which an incorrect digit was included in the list (incorrect condition) (Lefebvre et al., 2005; Marchand et al., 2006). The findings showed a positive P2 and P3 in the correct condition, and conversely showed a prolonged positive slow wave for the incorrect condition; this suggests that the two conditions are associated with different patterns of activation. Another study compared forward and backward recall, showing the presence of high negative correlations between P3 latency and the DST (Walhovd and Fjell, 2002). Finally, research has shown that the amplitudes of the P3a and P3b ERPs are reduced during backward recall in verbal but not in visuospatial tasks (Nulsen et al., 2010). This finding, suggesting a different pattern 


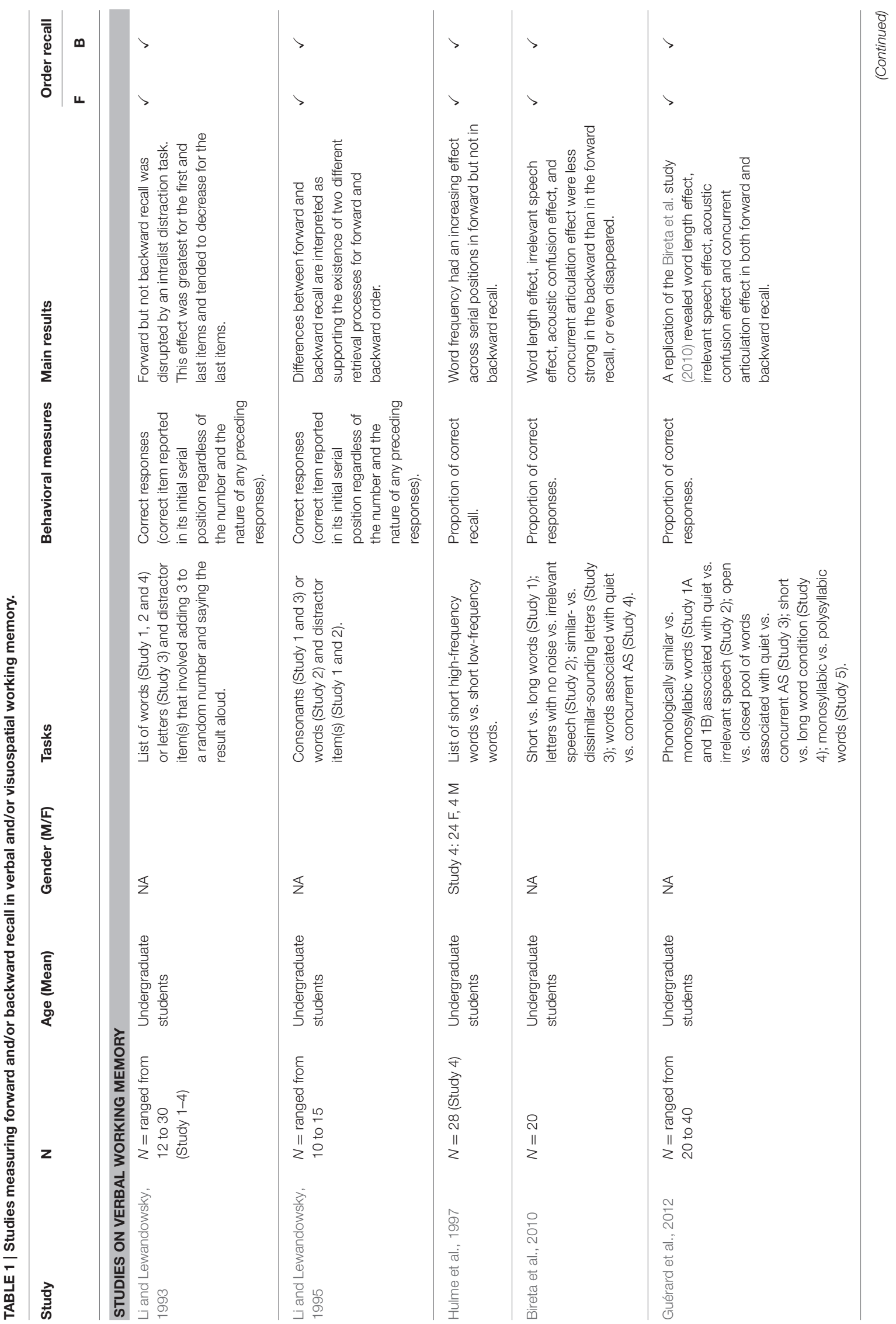




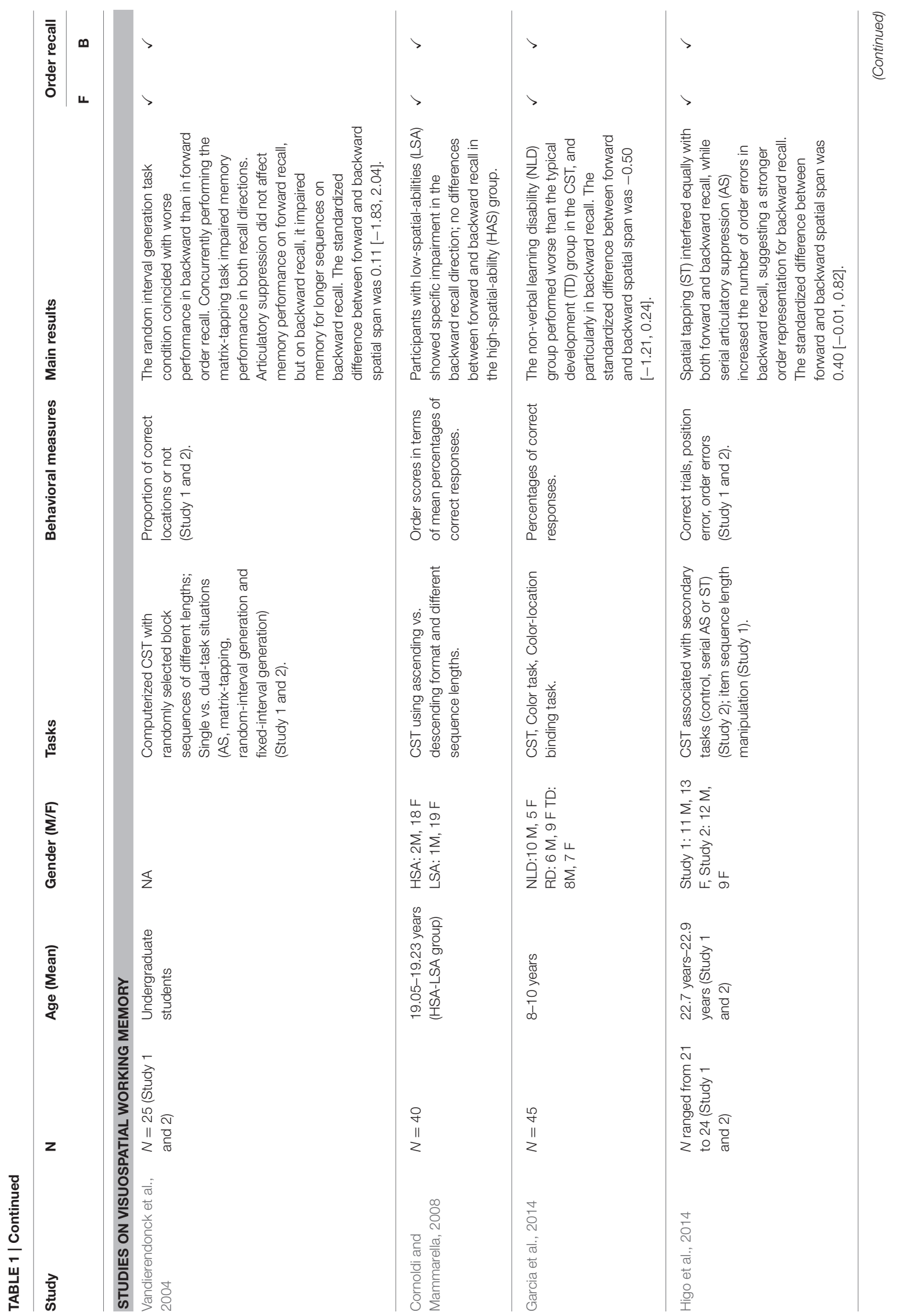




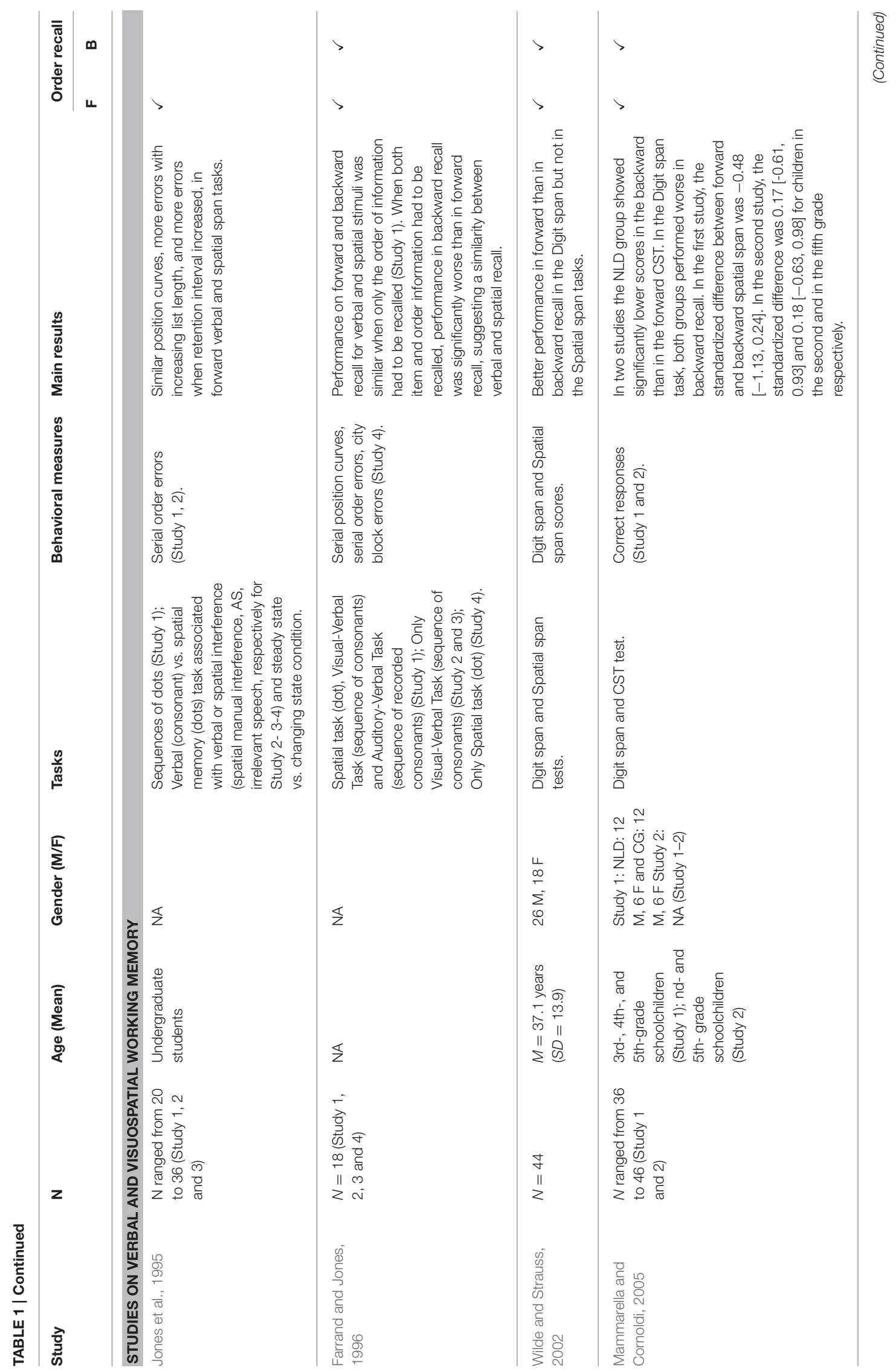




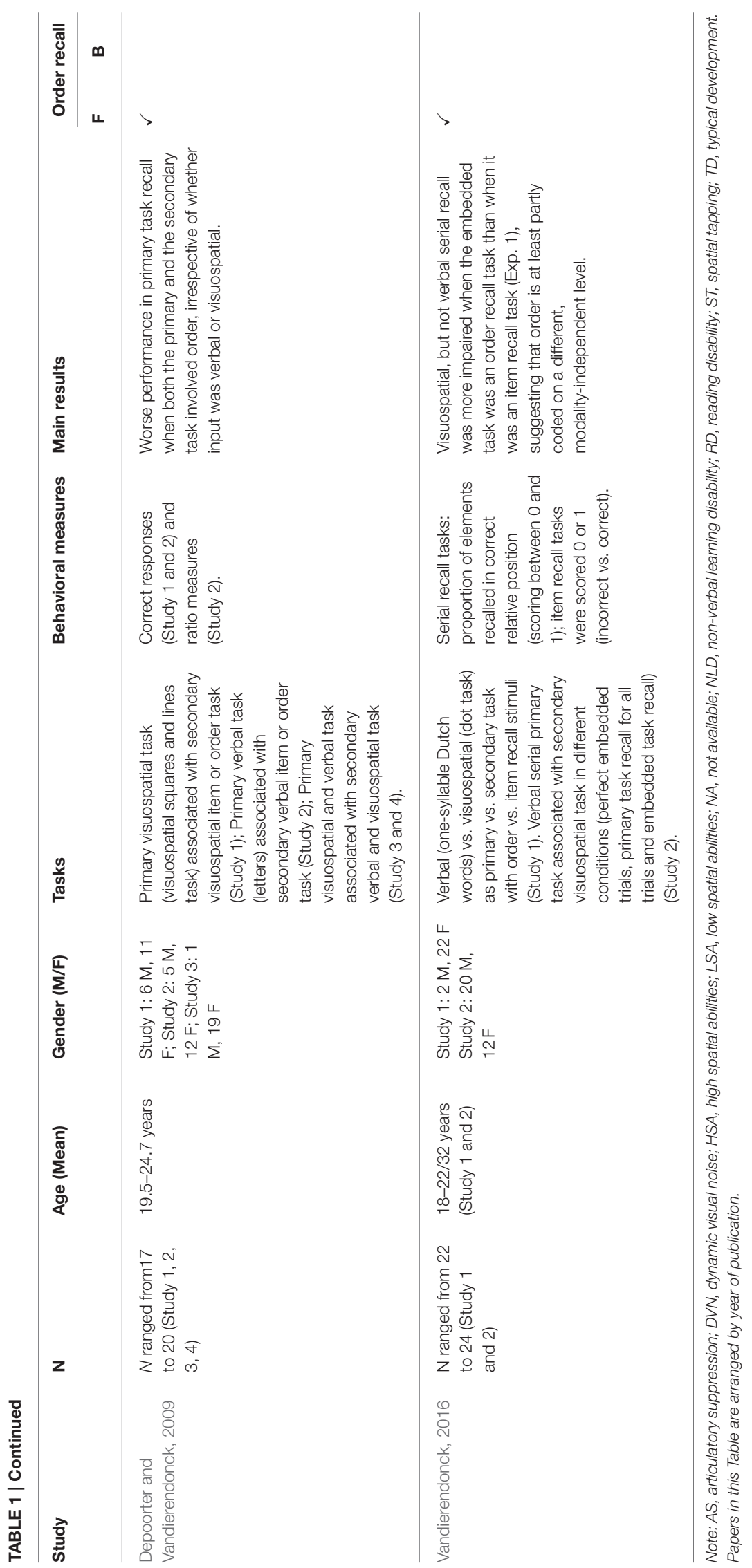




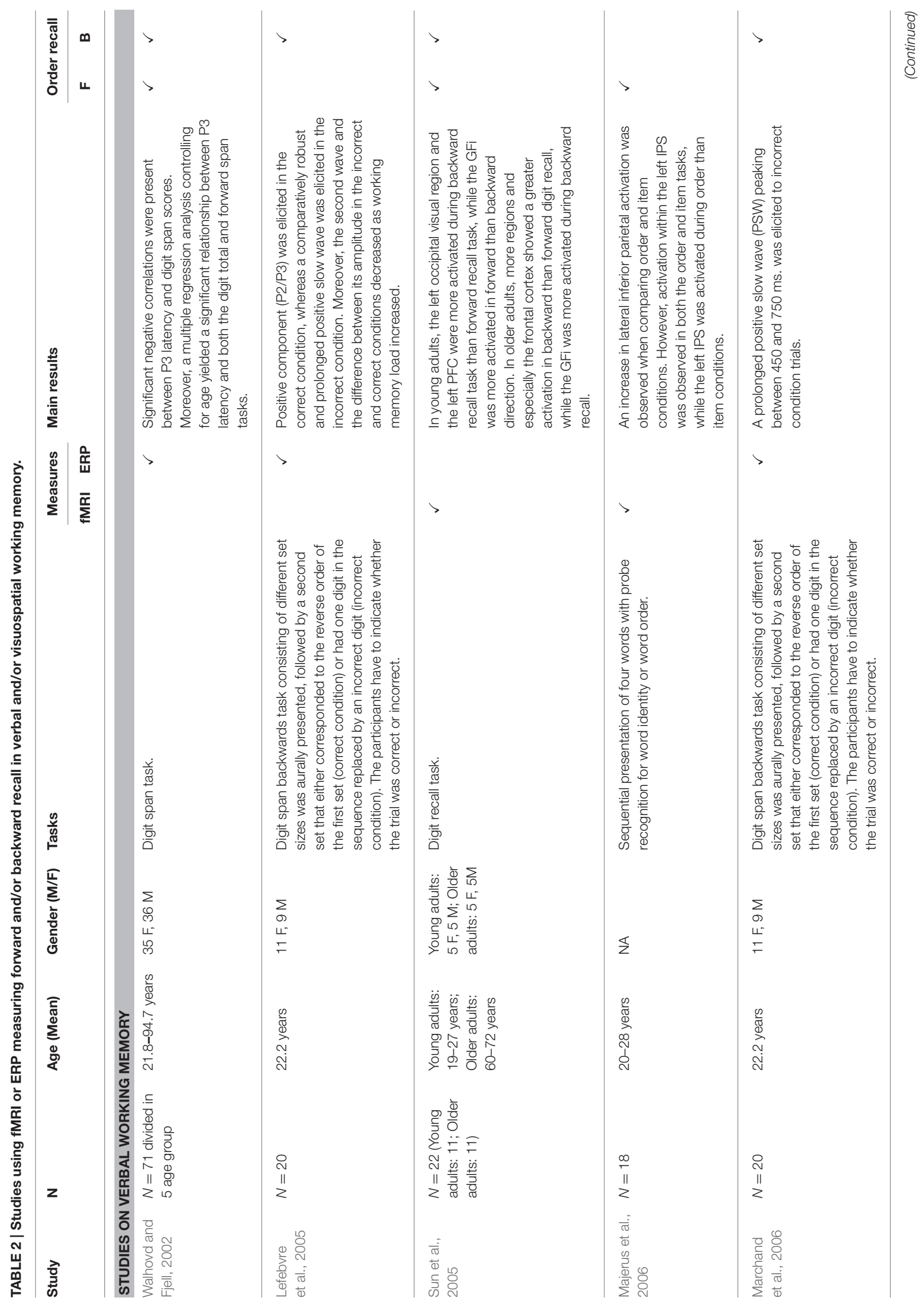




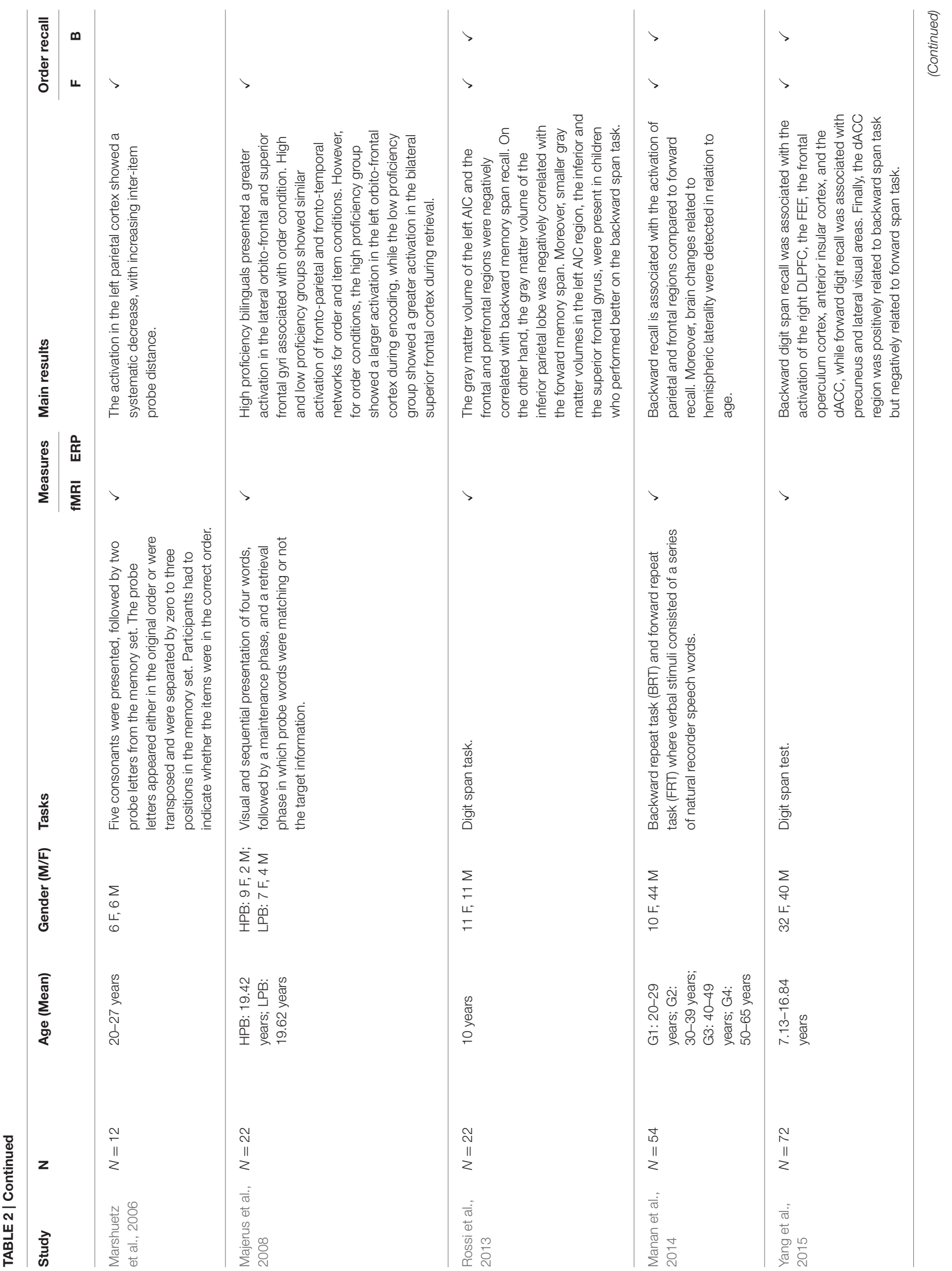




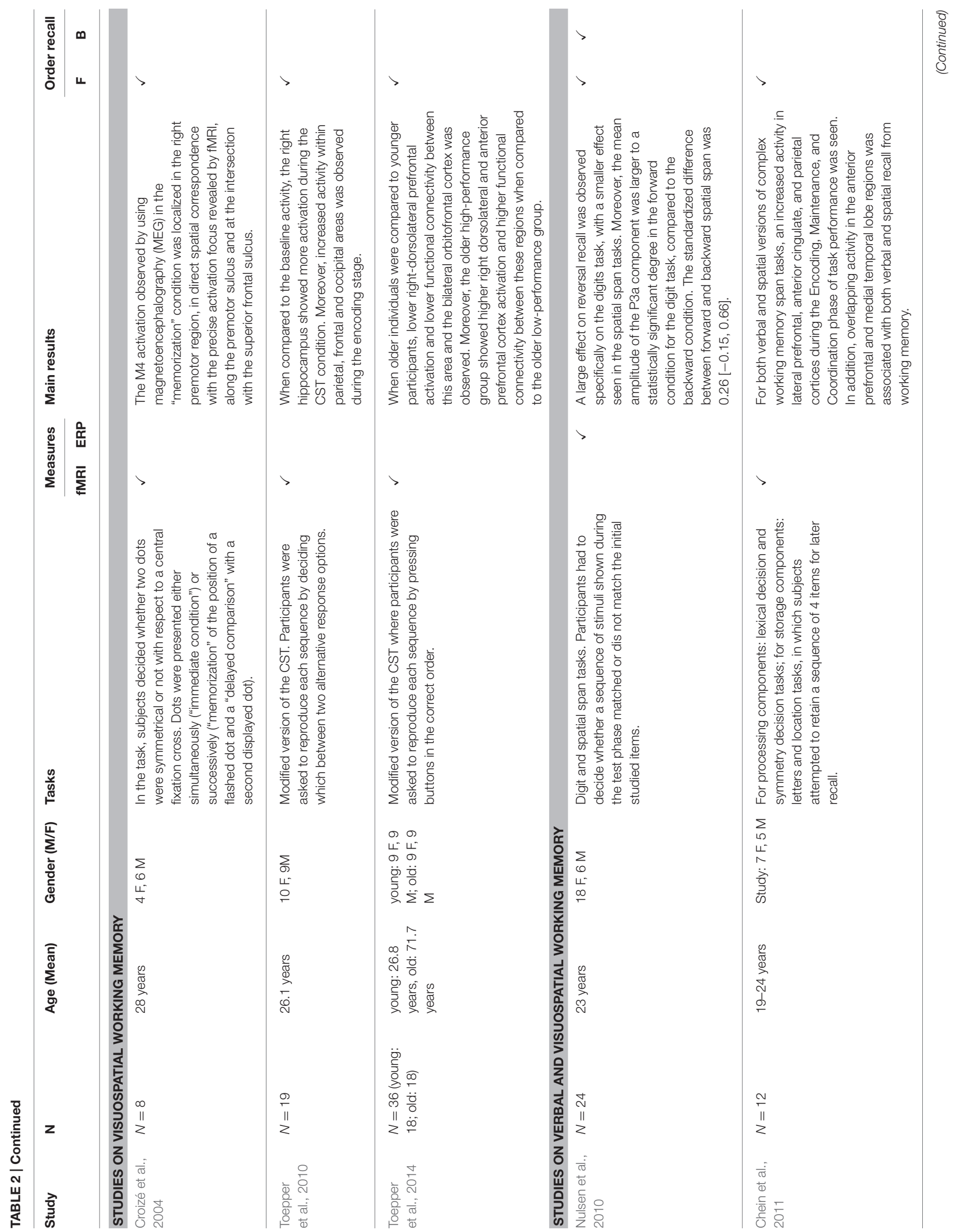




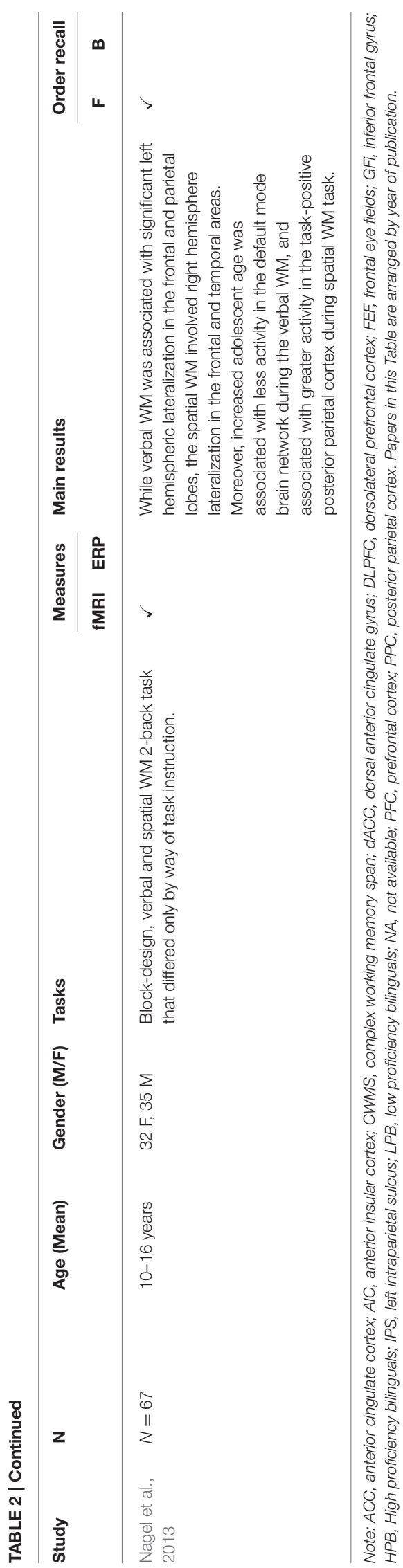

of activation between verbal and spatial backward spans, seems to indicate a reduction of attentional resources in the verbal backward span (see Nulsen et al., 2010).

As for fMRI studies, in the verbal domain, two studies compared the recognition of ordered and item information. In the ordered condition, results show a greater bilateral activation in the intraparietal sulcus (IPS) and in the premotor frontal areas (Henson et al., 2000; Marshuetz et al., 2000), supporting the idea that ordered material requires more attentional resources. However, a study by Majerus et al. (2006) failed to find a consistent differential activation in the left IPS, indicating that the difference between order and item condition is related to a specific network that links the left and right IPS with the right dorsal premotor cortex and the superior cerebellum. Interestingly, bilingual individuals with a high level of proficiency in both languages demonstrated greater activation in the lateral orbito-frontal region and in the superior frontal gyri associated with the updating of ordered information (Majerus et al., 2008), confirming the presence of different patterns of activation for order and item encoding.

Studies comparing different patterns of activation in forward and backward recall are mainly focused on verbal material. Research has shown the involvement of different neural correlates in forward and backward recall of digits (Manan et al., 2014). Another study showed that backward digit recall was associated with a higher activation of the left occipital visual region and the left prefrontal cortex (PFC) in young adults (Sun et al., 2005), supporting the idea of the involvement of visuospatial processing during backward verbal tasks (e.g., Larrabee and Kane, 1986; Hoshi et al., 2000). Moreover, young adults showed a greater activation in the inferior frontal gyrus in both forward and backward recall. The activation was associated with a limited overlap, providing evidence in favor of a distinction between forward and backward recall activation patterns (Sun et al., 2005). Furthermore, the central executive seems to be highly taxed during backward digit recall (Carlesimo et al., 1994). These results are in line with previous findings suggesting that the backward DST was associated with the activation of regions that are also involved in tasks requiring high cognitive control. Such activated regions include the right dorsolateral PFC, the frontal eye field, the frontal operculum cortex, the anterior insular cortex and the dorsal anterior cingulate cortex (dACC) (Yang et al., 2015). Intriguingly, activation of the dACC region was positively related to the backward span task but negatively related to the forward one (Yang et al., 2015). Finally, results with child subjects revealed the presence of distinct negative correlations between the forward/backward DST and the gray matter volume of some brain areas, such as the left AIC region, the inferior frontal gyrus and the superior frontal gyrus (Rossi et al., 2013).

While research on verbal material is plentiful in the literature, fMRI studies on the visuospatial domain are mainly focused on the forward span. In a study, when participants were asked to decide whether two dots were symmetrical or not, the results revealed that in the memorization condition, where participants had to judge whether the symmetry of the second dot's position related to the memorized position of the first dot, the right 
premotor region was activated (Croizé et al., 2004). Two other studies involved a modified version of the CST, and showed the involvement of the hippocampus in the encoding of spatial locations (Toepper et al., 2010). In addition, age effects were observed in the right-dorsolateral prefrontal cortex, which was found to be less activated in the older group compared to the younger one (Toepper et al., 2014).

Finally, in two studies comparing verbal and visuospatial domains the results seemed to favor a distinction between the two domains (Chein et al., 2011; Nagel et al., 2013). For example, Nagel et al. (2013) considered the difference between the verbal and visuospatial domains from a developmental point of view, suggesting that increased adolescent age was associated with less activity in the default mode brain network (i.e., a brain network more commonly active at rest and deactivated during task) during a verbal WM task. In contrast, increased adolescent age was associated with greater activity in the posterior parietal cortex during a spatial WM task.

\section{Implications for the Working Memory Models}

The presence of different results for order recall in verbal and visuospatial domains is considered as evidence in support of several existing theoretical models of WM. Baddeley's WM model postulates the existence of two domain-specific subsystems involved in the storage of verbal and visuospatial information: the phonological loop and the visuospatial sketchpad, respectively. These two components are linked with the central executive system that integrates and manipulates information (e.g., Baddeley, 1986). In this model, the phonological loop explains several phenomena affecting serial recall in verbal STM, such as the influence of word length, articulatory suppression, phonological similarity and item similarity. The decline in performance in the backward span is also interpreted in relation to the central executive's taxed resources (Baddeley, 1986). However, a limit of this model is that it fails to explain the results observed in visuospatial tasks and lacks a clear distinction between recalling sequential ordered information, and recalling unordered information.

Alternative models of WM propose a modality-independent view, with no distinction between verbal and visuospatial input. This approach is supported by the similar serial position curves detected in the verbal and visuospatial domains, and the shared memory resources for maintaining information in a given order (Engle, 1996; Cowan, 1999, 2005; Oberauer, 2009). It has also been suggested that the difference between verbal and visuospatial span tasks in forward and backward directions is associated with dissimilar retrieval demands: while participants use blocks to give their answers in the CST, in the DST the digits are not presented during the retrieval phase. Thus, verbal tasks would seem to require the recall of both items and order information, while visuospatial tasks would only require the latter (Farrand and Jones, 1996). Similarities between serial order and position effects in the verbal and spatial domains can be explained by assuming that order is treated similarly across different domains (Smyth, 1996). A comparable view is based on the assumption that there is a modality-independent process for serial order retention, and a domain-specific process for item retention (Depoorter and Vandierendonck, 2009).

Another hypothesis postulates that verbal and visuospatial forward serial recall measures the "passive" STM component, while backward recall involves executive control resources (Carlesimo et al., 1994; Hester et al., 2004). Developmental studies, combined with research in which clinical samples were considered, have helped to clarify this hypothesis. For example, a greater involvement of executive control in backward serial recall has been demonstrated in typically-developing children (Alloway et al., 2009), and in children with ADHD or learning disabilities (Cornoldi et al., 2013a,b; Giofrè et al., 2016), but not in adults (Rosen and Engle, 1997).

Concerning the visuospatial domain, a model has been proposed (Logie, 1995; Darling et al., 2007) that distinguishes between the visual cache, linked with the temporary storage of static visual information, and the inner scribe, involved in the dynamic processing of sequences of movement. According to this model, the maintenance of sequential information is crucial in spatial processes. This is in contrast with other models which are based on the assumption that visuospatial processes tend to lose sequential information in favor of simultaneously presented information (Paivio, 1971).

Finally, a model distinguishing between a visual component and two spatial subcomponents involving spatial-sequential and spatial-simultaneous processes has been proposed (Lecerf and De Ribaupierre, 2005; Mammarella et al., 2008, 2013). This is supported by findings in different groups of children with developmental disorders (Mammarella et al., 2003, 2006; Lanfranchi et al., 2015), and in healthy adults (Mammarella et al., 2013). In this view, Mammarella and Cornoldi (2005) suggested that differences in forward and backward spatial recall are due not only to the involvement of the executive control, but also to involvement of spatial-sequential and spatial-simultaneous processing. Research has also shown that backward recall in the CST requires less executive control and more spatial processing, supporting the idea that backward recall involves a modalityindependent order coding system (Higo et al., 2014). This hypothesis is supported by evidence suggesting that the backward CST involves both visuospatial processing and executive control (Vandierendonck et al., 2004; Vandierendonck, 2016).

We decided to investigate this result further by analyzing papers including both versions of the CST. Nine papers included in this review deal with both versions of the spatial span. Among these studies, two did not report means or effect sizes, making it impossible to calculate effect sizes (Farrand and Jones, 1996; Farrand et al., 2001); one reported data from a clinical sample (Wilde and Strauss, 2002), and one compared participants with high vs. low spatial abilities (Cornoldi and Mammarella, 2008). However, five studies reported descriptive statistics (i.e., Vandierendonck et al., 2004; Mammarella and Cornoldi, 2005; Nulsen et al., 2010; Garcia et al., 2014; Higo et al., 2014) and seven effect sizes were extracted from these studies (see also Tables 1, 2). When considering these effects together, and assuming random effects, the overall effect is $d_{\text {unb }}=0.039$ [-0.20, 0.28]. This finding seems to indicate the difference between forward 
and backward spatial span is very small and not statistically significant.

\section{CONCLUSIONS}

In the present paper, we reviewed findings on forward and backward recall in the verbal and visuospatial domains, considering the contribution of experimental and neuroscience studies.

The evidence from the cognitive studies is quite clear. Regarding the verbal domain, the verbal recall task is often characterized by a clear difference between the forward and the backward version of the span, with lower performance in the latter. In the visuospatial domain-at least when typically developing children or healthy adults are considered-it is more difficult to detect differences between recall of the forward and backward versions of the task.

Overall, experimental studies do not provide a clear support for any theoretical model described above. Advances in technical and quantitative methods of neuroscience over the past years have aided and propelled analysis in various fields of psychology. Neuroscientific studies cited in this review have indicated that verbal recall, in the backward order in particular, seems to require greater cognitive resources (Manan et al., 2014). In addition, different brain areas are activated in verbal and visuospatial tasks (Sun et al., 2005; Yang et al., 2015). These findings support modality independent models of WM, and in fact verbal performance and visuospatial performance is always clearly distinguishable.

Unfortunately, to-date no study has compared forward and backward recall in verbal and visuospatial domains in relation to neural correlates. A promising future line of research would involve studies that examine the simultaneous storage of information derived from different modalities. In fact, future efforts should directly compare the neural correlates of forward

\section{REFERENCES}

Alloway, T. P., Gathercole, S. E., Kirkwood, H., and Elliott, J. (2009). The cognitive and behavioral characteristics of children with low working memory. Child Dev. 80, 606-621. doi: 10.1111/j.1467-8624.2009.01282.x

Baddeley, A. (1986). Working Memory. Oxford: Clarendon Press.

Baddeley, A. D., and Hitch, G. J. (1974). "Working memory," in The Psychology of Learning and Motivation Vol. 8., ed G. H. Bower (New York, NY: Academic Press), 47-89.

Berch, D. B., Krikorian, R., and Huha, E. M. (1998). The Corsi-block tapping task: methodological and theoretical considerations. Brain Cogn. 38, 317-338. doi: 10.1006/brcg.1998.1039

Bireta, T. J., Fry, S. E., Jalbert, A., Neath, I., Surprenant, A. M., Tehan, G., et al. (2010). Backward recall and benchmark effects of working memory. Mem. Cognit. 38, 279-291. doi: 10.3758/MC.38.3.279

Carlesimo, G. A., Fadda, L., Lorusso, S., and Caltagirone, C. (1994). Verbal and spatial memory spans in Alzheimer's and multi-infarct dementia. Acta Neurol. Scand. 89, 132-138. doi: 10.1111/j.1600-0404.1994. tb01648.x

Chein, J. M., Moore, A. B., and Conway, A. R. (2011). Domain-general mechanisms of complex working memory span. Neuroimage 54, 550-559. doi: 10.1016/j.neuroimage.2010.07.067 and backward recall in verbal and visuospatial domains, and do so within a single study. Furthermore, other techniques should be used in order to collect further evidence. Potentially, future studies could employ other psycho-physiological measures such as eye movements, or neuroimaging techniques such as transcranial magnetic stimulation or magneto-encephalography. These kinds of additional analytical measures could allow researchers to reach clearer results. Moreover, methodologies and the types of tasks used in future studies should be consistent and comparable.

Ultimately, only few developmental studies have been carried out to-date; therefore, how the serial recall of verbal and spatial information develops is not yet completely clear. A deeper understanding of such changes could in turn help in improving our understanding of currently existing theoretical models.

Despite some shortcomings, the findings collectively gathered in this review are both comprehensive and beneficial to those currently researching in this field. The take home messages from these reviews are as follows: (1) verbal and spatial WM modalities seem to be distinct; (2) there is overwhelming evidence for a distinction between the forward and backward digit span; and (3) there is no clear evidence for a distinction between forward and backward spatial span.

\section{AUTHOR CONTRIBUTIONS}

ED, ICM, and DG contributed to the study concept and design. ED, ICM, and DG wrote the paper. All authors approved the final version of the manuscript for submission.

\section{ACKNOWLEDGMENTS}

We would like to thank David Recine for his editing and helpful comments.

Cornoldi, C., Giofrè, D., Calgaro, G., and Stupiggia, C. (2013a). Attentional WM is not necessarily specifically related with fluid intelligence: the case of smart children with ADHD symptoms. Psychol. Res. 77, 508-515. doi: $10.1007 / \mathrm{s} 00426-012-0446-8$

Cornoldi, C., and Mammarella, I. C. (2008). A comparison of backward and forward spatial spans. Q. J. Exp. Psychol. 61, 674-682. doi: 10.1080/17470210701774200

Cornoldi, C., Orsini, A., Cianci, L., Giofrè, D., and Pezzuti, L. (2013b). Intelligence and working memory control: evidence from the WISCIV administration to Italian children. Learn. Individ. Differ. 26, 9-14. doi: 10.1016/j.lindif.2013.04.005

Cornoldi, C., Venneri, A., Marconato, F., Molin, A., and Montinari, C. (2003). A rapid screening measure for the identification of visuospatial learning disability in schools. J. Learn. Disabil. 36, 299-306. doi: 10.1177/002221940303 60040201

Cowan, N. (1999). "An embedded-processes model of working memory," in Models of Working Memory: Mechanisms of Active Maintenance and Control, eds A. Miyake and P. Shah (Cambridge: Cambridge University Press), 62-101.

Cowan, N. (2005). Working Memory Capacity. Hove: Psychology Press.

Croizé, A. C., Ragot, R., Garnero, L., Ducorps, A., Pelegrini-Issac, M., Dauchot, K., et al. (2004). Dynamics of parietofrontal networks underlying visuospatial short-term memory encoding. Neuroimage 23, 787-799. 
Darling, S., Della Sala, S., and Logie, R. H. (2007). Behavioural evidence for separating components within visuo-spatial working memory. Cogn. Process. 8, 175-181. doi: 10.1007/s10339-007-0171-1

Depoorter, A., and Vandierendonck, A. (2009). Evidence for modalityindependent order coding in working memory. Q. J. Exp. Psychol. 62, 531-549. doi: 10.1080/17470210801995002

Engle, R. W. (1996). "Working memory and retrieval: an inhibition-resource approach," in Working Memory and Human Cognition, ed J. T. E. Richardson (Oxford: Oxford University Press), 89-119.

Farrand, P., and Jones, D. M. (1996). Direction of report in spatial and verbal serial short-term memory. Q. J. Exp. Psychol. A 49, 140-158.

Farrand, P., Parmentier, F. B. R., and Jones, D. M. (2001). Temporal-spatial memory: retrieval of spatial information does not reduce recency. Acta Psychol. 106, 285-301. doi: 10.1016/S0001-6918(00)00054-8

Garcia, R. B., Mammarella, I. C., Tripodi, D., and Cornoldi, C. (2014). Visuospatial working memory for locations, colours, and binding in typically developing children and in children with dyslexia and non-verbal learning disability. $\mathrm{Br}$. J. Dev. Psychol. 32, 17-33. doi: 10.1111/bjdp.12019

Giofrè, D., Stoppa, E., Ferioli, P., Pezzuti, L., and Cornoldi, C. (2016). Forward and backward digit span difficulties in children with specific learning disorder. J. Clin. Exp. Neuropsychol. 38, 478-486. doi: 10.1080/13803395.2015.1125454

Guérard, K., Saint-Aubin, J., Burns, S. C., and Chamberland, C. (2012). Revisiting backward recall and benchmark memory effect: a reply to Bireta et al. (2010). Mem. Cognit. 40, 388-407. doi: 10.3758/s13421-011-0156-2

Henson, R. N., Burgess, N., and Frith, C. D. (2000). Recoding, storage, rehearsal, and grouping in verbal short-term memory: an fMRI study. Neuropsychologia 38, 426- 440. doi: 10.1016/S0028-3932(99)00098-6

Hester, R. L., Kinsella, G. J., and Ong, B. (2004). Effect of age on forward and backward span tasks. J. Int. Neuropsychol. Soc. 10, 475-481. doi: $10.1017 /$ S1355617704104037

Higo, K., Minamoto, T., Ikeda, T., and Osaka, M. (2014). Robust order representation is required for backward recall in the Corsi blocks task. Front. Psychol. 5:1285. doi: 10.3389/fpsyg.2014.01285

Hoshi, Y., Oda, I., Wada, Y., Ito, Y., Yamashita, Y., Oda, M., et. al. (2000). Visuospatial imagery is a fruitful strategy for the digit span backward task: a study with near-infrared optical tomography. Cogn. Brain Res. 9, 339-342. doi: 10.1016/S0926-6410(00)00006-9

Hulme, C., Roodenrys, S., Schweickert, R., Brown, G. D. A., Martin, S., and Stuart, G. (1997). Word frequency effects on short-term memory tasks: evidence for a reintegration process in immediate serial recall. J. Exp. Psychol. Learn. Mem. Cogn. 23, 1217-1232. doi: 10.1037/0278-7393.23.5.1217

Jones, D., Farrand, P., Stuart, G., and Morris, N. (1995). Functional equivalence of verbal and spatial information in serial short-term memory. J. Exp. Psychol. Learn. Mem. Cogn. 21:1008. doi: 10.1037/0278-7393.21.4.1008

Lanfranchi, S., De Mori, L., Mammarella, I. C., Carretti, B., and Vianello, R. (2015). Spatial-sequential and spatial-simultaneous working memory in individuals with William syndrome. Am. J. Intellect. Dev. Disabil. 120, 193-202. doi: 10.1352/1944-7558-120.3.193

Larrabee, G. J., and Kane, R. L. (1986). Reversed digit repetition involves visual and verbal processes. Int. J. Neurosci. 30, 11-15. doi: 10.3109/00207458608985649

Lecerf, T., and De Ribaupierre, A. (2005). Recognition in a visuospatial memory task: the effect of presentation. Eur. J. Cogn. Psychol. 17, 47-75. doi: 10.1080/09541440340000420

Lefebvre, C. D., Marchand, Y., Eskes, G. A., and Connolly, J. F. (2005). Assessment of working memory abilities using an event-related brain potential (ERP)compatible digit span backward task. Clin. Neurophysiol. 116, 1665-1680. doi: 10.1016/j.clinph.2005.03.015

Li, S.-C., and Lewandowsky, S. (1993). Intralist distracters and recall direction: constraints on models of memory for serial order. J. Exp. Psychol. Learn. Mem. Cogn. 19, 895-908. doi: 10.1037/0278-7393.19.4.895

Li, S.-C., and Lewandowsky, S. (1995). Forward and backward recall: different retrieval processes. J. Exp. Psychol. Learn. Mem. Cogn. 21, 837-847. doi: 10.1037/0278-7393.21.4.837

Logie, R. H. (1995). Visuo-Spatial Working Memory. Hove: Erlbaum.

Logie, R. H., Saito, S., Morita, A., Varma, S., and Norris, D. (2016). Recalling visual serial order for verbal sequences. Mem. Cognit. 44, 590-607.

Majerus, S., Belayachi, S., De Smedt, B., Leclercq, A. L., Martinez, T., Schmidt, C., et al. (2008). Neural networks for short-term memory for order differentiate high and low proficiency bilinguals. Neuroimage 42, 1698-1713. doi: 10.1016/j.neuroimage.2008.06.003

Majerus, S., Poncelet, M., Van der Linden, M., Albouy, G., Salmon, E., Sterpenich, V., et al. (2006). The left intraparietal sulcus and verbal shortterm memory: focus of attention or serial order?. Neuroimage 32, 880-891. doi: 10.1016/j.neuroimage.2006.03.048

Mammarella, I. C., Borella, E., Pastore, M., and Pazzaglia, F. (2013). The structure of visuospatial memory in adulthood. Learn. Individ. Differ. 25, 99-110. doi: 10.1016/j.lindif.2013.01.014

Mammarella, I. C., and Cornoldi, C. (2005). Sequence and space: the critical role of a backward spatial span in the working memory deficit of visuospatial learning disabled children. Cogn. Neuropsychol. 22, 1055-1068. doi: 10.1080/02643290442000509

Mammarella, I. C., Cornoldi, C., Pazzaglia, F., Toso, C., Grimoldi, M., and Vio, C. (2006). Evidence for a double dissociation between spatial-simultaneous and spatial-sequential working memory in visuospatial (nonverbal) learning disabled children. Brain Cogn. 62, 58-67. doi: 10.1016/j.bandc.2006. 03.007

Mammarella, I. C., Pazzaglia, F., and Cornoldi, C. (2008). Evidence for different components in children's visuospatial working memory. Br. J. Dev. Psychol. 26, 337-355. doi: 10.1348/026151007X236061

Mammarella, N., Cornoldi, C., and Donadello, E. (2003). Visual but not spatial working memory deficit in children with spina bifida. Brain Cogn. 53, 311-314. doi: 10.1016/S0278-2626(03)00132-5

Manan, H. A., Franz, E. A., Yusoff, A. N., and Mukari, S. Z. M. S. (2014). Age-related brain activation during forward and backward verbal memory tasks. Neurol. Psychiatry Brain Res. 20, 76-86. doi: 10.1016/j.npbr.2014. 08.001

Marchand, Y., Lefebvre, C. D., and Connolly, J. F. (2006). Correlating digit span performance and event-related potentials to assess working memory. Int. J. Psychophysiol. 62, 280-289. doi: 10.1016/j.ijpsycho.2006.05.007

Marshuetz, C., Reuter-Lorenz, P. A., Smith, E. E., Jonides, J., and Noll, D. C. (2006). Working memory for order and the parietal cortex: an event-related functional magnetic resonance imaging study. Neuroscience 139, 311-316.

Marshuetz, C., Smith, E. E., Jonides, J., DeGutis, J., and Chenevert, T. L. (2000). Order information in working memory: fMRI evidence for parietal and prefrontal mechanisms. J. Cogn. Neurosci. 12, 130-144. doi: 10.1162/08989290051137459

Nagel, B. J., Herting, M. M., Maxwell, E. C., Bruno, R., and Fair, D. (2013). Hemispheric lateralization of verbal and spatial working memory during adolescence. Brain Cogn. 82, 58-68. doi: 10.1016/j.bandc.2013. 02.007

Nulsen, C. E., Fox, A. M., and Hammond, G. R. (2010). Processes contributing to forward and backward span: an ERP investigation. Neuroreport 21, 298-302. doi: 10.1097/WNR.0b013e32833730f0

Oberauer, K. (2009). "Design for a working memory," in The Psychology of Learning and Motivation Vol. 51B., ed H. Ross (London: Academic Press), 45-100.

Paivio, A. (1971). Imagery and Verbal Processes. New York, NY: Holt, Reinhart \& Winston.

Penney, C. G. (1989). Modality effects and the structure of short-term verbal memory. Mem. Cognit. 17, 398-422. doi: 10.3758/BF03202613

Ritchie, G., Tolan, G. A., Tehan, G., Goh, H. E., Guérard, K., and SaintAubin, J. (2015). Phonological effects in forward and backward serial recall: qualitative and quantitative differences. Can. J. Exp. Psychol. 69:95. doi: $10.1037 /$ cep0000028

Rosen, V. M., and Engle, R. W. (1997). Forward and backward serial recall. Intelligence 25, 37-47. doi: 10.1016/S0160-2896(97)90006-4

Rossi, S., Lubin, A., Simon, G., Lanoë, C., Poirel, N., Cachia, A., et al. (2013). Structural brain correlates of executive engagement in working memory: children's inter-individual differences are reflected in the anterior insular cortex. Neuropsychologia 51, 1145-1150. doi: 10.1016/j.neuropsychologia.2013.03.011

Smyth, M. M. (1996). Serial order in spatial immediate memory. Q. J. Exp. Psychol. A 49, 159-177. doi: 10.1080/713755615

Sun, X., Zhang, X., Chen, X., Zhang, P., Bao, M., Zhang, D., et al. (2005). Age-dependent brain activation during forward and backward digit recall revealed by fMRI. Neuroimage 26, 36-47. doi: 10.1016/j.neuroimage.2005. 01.022 
Toepper, M., Markowitsch, H. J., Gebhardt, H., Beblo, T., Bauer, E., Woermann, F. G., et al. (2014). The impact of age on prefrontal cortex integrity during spatial working memory retrieval. Neuropsychologia 59, 157-168. doi: 10.1016/j.neuropsychologia.2014.04.020

Toepper, M., Markowitsch, H. J., Gebhardt, H., Beblo, T., Thomas, C., Gallhofer, B., et al. (2010). Hippocampal involvement in working memory encoding of changing locations: an fMRI study. Brain Res. 1354, 91-99. doi: 10.1016/j.brainres.2010.07.065

Vandierendonck, A. (2016). Modality independence of order coding in working memory: evidence from cross-modal order interference at recall. Q. J. Exp. Psychol. 69, 161-179. doi: 10.1080/17470218.2015. 1032987

Vandierendonck, A., Kemps, E., Fastame, M. C., and Szmalec, A. (2004). Working memory components of the Corsi block task. Br. J. Psychol. 95, 57-79. doi: $10.1348 / 000712604322779460$

Walhovd, K. B., and Fjell, A. M. (2002). The relationship between P3 and neuropsychological function in an adult life span sample. Biol. Psychol. 62, 65-87. doi: 10.1016/S0301-0511(02)00093-5
Wilde, N., and Strauss, E. (2002). Functional equivalence of WAIS-III/WMS-III digit and spatial span under forward and backward recall conditions. Clin. Neuropsychol. 16, 322-330. doi: 10.1076/clin.16.3.322.13858

Yang, Z., Jutagir, D. R., Koyama, M. S., Craddock, R. C., Yan, C. G., Shehzad, Z., et al. (2015). Intrinsic brain indices of verbal working memory capacity in children and adolescents. Dev. Cogn. Neurosci. 15, 67-82. doi: 10.1016/j.den.2015.07.007

Conflict of Interest Statement: The authors declare that the research was conducted in the absence of any commercial or financial relationships that could be construed as a potential conflict of interest.

Copyright (๑) 2017 Donolato, Giofrè and Mammarella. This is an open-access article distributed under the terms of the Creative Commons Attribution License (CC BY). The use, distribution or reproduction in other forums is permitted, provided the original author(s) or licensor are credited and that the original publication in this journal is cited, in accordance with accepted academic practice. No use, distribution or reproduction is permitted which does not comply with these terms. 